\title{
Enhancement of Cooling Effectiveness with Mist Assisted Film Cooling
}

\author{
Pratibha Biswal $^{1}$, Udaydeep Jindal ${ }^{2}$, Sathi Rajesh Reddy ${ }^{2}$ \\ ${ }^{1}$ Department of Chemical Engineering, Shiv Nadar University \\ Dadri, UP, India \\ pratibha.biswal@snu.edu.in \\ ${ }^{2}$ Department of Mechanical Engineering, Shiv Nadar University \\ Dadri, UP, India
}

\begin{abstract}
Film cooling is a well known technique used to cool the gas turbine blades from hot combustion gases. In this study, a more prominent technique called mist assisted film cooling is numerically investigated. Water droplets are injected in to the cooling air to enhance the cooling performance. The cooling performance is investigated downstream the film cooling hole in a straight channel. The coolant jet (dry air) is supplied via the film cooling hole along with water droplets injected at same velocity and temperature. The computational simulations are performed using the commercial software ANSYS FLUENT 17.2. As the concentration difference exists between the droplet surface and bulk air, the evaporation takes place and before reaching to the mainstream, the water droplets vaporize to form water vapors. Thus, the temperature of the coolant further reaches a lesser temperature. This phenomenon does not occur when the coolant is pure dry air and this part is addressed for the first time in this study. Results showed that the overall cooling effectiveness is always larger for the mist-air case compared to that for the pure dry air case. At a specific mass flow rate and specific mist percentage, the optimum angle of inlet coolant is found to be 35 degrees. Also, at a specific mass flow rate and specific mist percentage, increase in the droplet diameter results in the decrease of the cooling effectiveness. It is also found that increasing the number of holes to two, increases the cooling effectiveness.
\end{abstract}

Keywords: Cooling Effectiveness, Mist, Turbine blade, Evaporative cooling, Film cooling.

\section{Introduction}

Film cooling of turbine blade consists of pumping the cooling air out of the blade through single or multiple small holes. A thin layer (the film) of cooling air is created on the external surface of the blade, reducing the heat transfer from main flow. The film cooling involving the injection of mist (small amount of liquid water droplets) into the cooling air can improve the cooling performance. As the water droplets are injected along with the coolant air, the concentration difference between the water droplets and the coolant air results in the evaporation of the former leading to drop in temperature and enhancement of the turbine blade cooling efficiency.

Many studies can be found in the literature that proves the effect of the mist injection on the cooling effectiveness. Guo et. al. [1-3] investigated various numerical and experimental studies for mist based cooling of straight tube involving highly superheated wall temperatures. Wang et al. [4] carried out a series of experiments to investigate the cooling of hot solid surface using mist for different initial surface temperatures, droplet size, and droplet velocity. Li and Wang [5-7] also numerically simulated air/mist film cooling. Wang and $\mathrm{Li}$ [8] numerically studied the enhancement of the cooling effectiveness with water mist injection involving various hole geometries at surface. Kim et al. [9] presented the evaporative cooling process involving moisture of air and small amount of water droplets using various simultaneous heat and mass transfer models. Dhanasekaran and Wang [10] performed computational simulations to investigate the mist cooling technique in a 180-degree tube bend at gas turbine working conditions. Computational study on the cooling enhancement of smooth and ribbed channels with various mist concentrations is also carried out by Dhanasekaran and Wang [11]. Jiang et al. [12] presented numerical simulations to understand film cooling enhancement on turbine vane involving injected mist along with the coolant air.

The main observation from the earlier works is the enhancement of the cooling effectiveness of gas turbine blades with the addition of mist to the coolant fluid. As mist film cooling is a promising technology and also as there are very less number of papers detailed analysis of the effect of the mist and coolant injection angle. Current study focuses on mist film cooling of a flat plate at various conditions of the coolant and mainstream. The effects of the coolant hole angle and droplet diameter 
are explained in detail based on the temperature contours and cooling effectiveness. In addition, this work presents the effect of multiple holes in the mist assisted cooling of a flat plate.

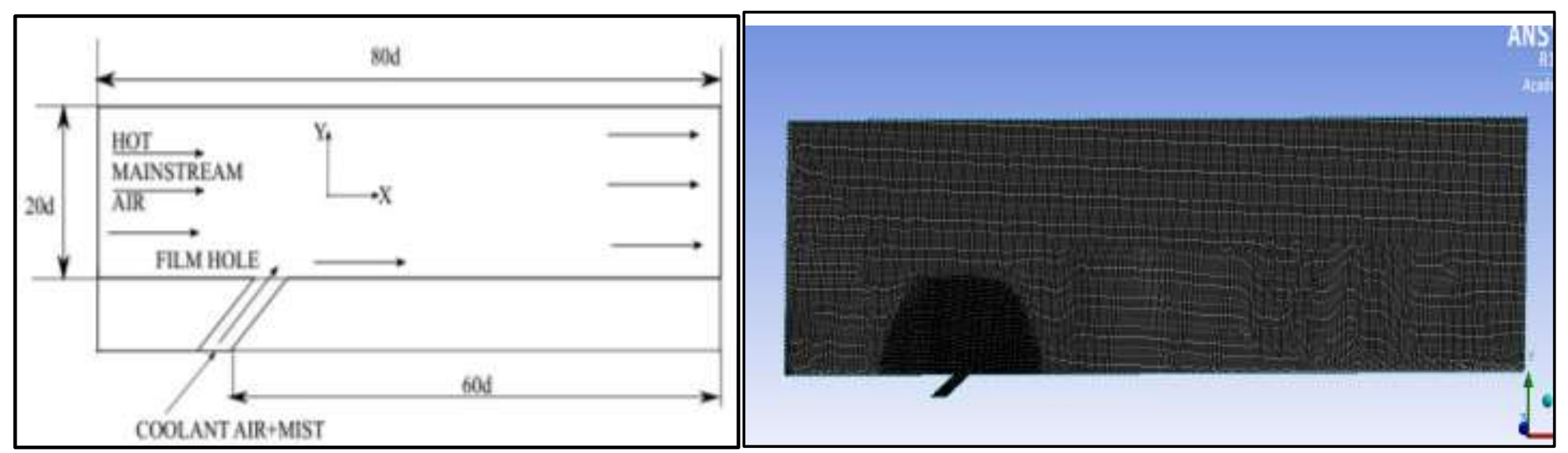

Fig. 1: The schematic diagram of the physical system (Left Panel) and computational unstructured mesh (Right Panel).

\section{Physical Model and governing equations}

The left panel of Fig. 1 illustrates the schematic diagram of geometric model and computational domain. The diameter of the coolant hole is $5 \mathrm{~mm}$, inclined at various angles with the mainstream direction. The mainstream flows over the flat plate and the dry coolant air flows through the coolant hole forming a film over the plate. The water droplets are injected at the inlet of the coolant hole along with the coolant air as shown in Fig. 1

The computational simulation is carried out based on the Eulerian-Lagrangian approach. The mainstream air and dry coolant air and water vapor are considered as element of the continuous (Eulerian) phase and the injected water droplets correspond to the dispersed or discrete (Lagrangian) phase. The Navier-Stokes equations are solved in the continuous phase whereas, each particle in the dispersed phase is tracked through the continuous flow field. The calculation for continuous and dispersed phases is coupled as exchange of mass, momentum and energy occurs between the continuous and dispersed phases.

The governing equations for the continuous phase are solved for certain number of iterations before solving the discrete phase equations. Taking this continuous phase variables as the base, one droplet per cell is injected and the droplet trajectories are calculated by applying discrete phase equations. Thus, the source terms for the continuous phase are estimated. The governing equations for continuous phase are again solved for another set of iterations with updated source terms. Thus the process of alternate solving of continuous and discrete phases continues till a converged solution is obtained. The governing equations are described in the following sections.

\subsection{Continuous phase (Air)}

The steady Navier-Stokes equations and equations for mass and energy are solved for the continuous phase in the considered problem. The governing equations for conservation of mass [Eq. 1], momentum [Eq. 2], and energy [Eq. 3] are given as:

$$
\begin{gathered}
\frac{\partial}{\partial x_{i}}\left(\rho u_{i}\right)=S_{m} \\
\frac{\partial}{\partial x_{i}}\left(\rho u_{i} u_{j}\right)=-\frac{\partial P}{\partial x_{j}}+\rho g_{j}+\frac{\partial \tau_{i j}}{\partial x_{j}}+F_{j} \\
\frac{\partial}{\partial x_{i}}\left(\rho C_{p} u_{i} T\right)=\frac{\partial}{\partial x_{j}}\left(\lambda_{e f f} \frac{\partial T}{\partial x_{i}}\right)+\mu \Phi+S_{h}
\end{gathered}
$$


where the source terms $S_{m}, F_{j}$, and $S_{h}$ are used to include the contributions from the dispersed phase. $\tau_{i j}$ is the symmetric stress tensor. $\mu \Phi$ is the heat of dissipation and $\lambda_{e f f}$ is the effective heat conductivity.

Since evaporation of droplets releases water vapor into the main airflow, species transport needs to be considered. There There are three species considered, water vapor, oxygen, and nitrogen. The equation for species transport is

$$
\frac{\partial}{\partial x_{i}}\left(\rho u_{i} C_{j}\right)=\frac{\partial}{\partial x_{j}}\left(\rho D_{e f f, j} \frac{\partial C_{j}}{\partial x_{i}}\right)+S_{j}
$$

where $C_{j}$ is the mass fraction of one of the species $j$ in the mixture, and $S_{j}$ is the source term for this species. $D_{e f f, j}$ is the effective diffusion coefficient considering the turbulence effect.

\subsection{Turbulence model}

The Reynolds number for the current flow conditions is of the order of $10^{4}$. Reference velocity scale is main stream velocity and length scale is channel height. Therefore, the flow is expected to be turbulent. Li and Wang [5] has mentioned that standard $\mathrm{k}-\epsilon$ model with enhanced wall treatment can predict the turbulent film cooling flows well. Hence the same turbulent model is used in the current study. Further details regarding turbulent model can be obtained in [5].

\subsection{Discrete Phase (Water Droplets)}

The droplets in the airflow can encounter inertia and hydrodynamic drags. Because of the forces experienced by a droplet in a flow field, the droplet can be either accelerated or decelerated. The velocity change can be formulated by

$$
m_{p} \frac{d v_{p}}{d t}=F_{d}+F_{g}+F_{o}
$$

where $m_{p}$ and $v_{p}$ are droplet mass and velocity respectively. $F_{d}$ is the drag of the fluid on the droplet and $F_{g}$ is the gravity. $F_{o}$ represents the other forceswhich includes the virtual mass force, thermophoretic force, Brownian force, and Saffman's lift force.

The inertia of the primary-phase mass encountered by the accelerating particles exerts a "virtual mass force" on the particles. Small particles suspended in a gas with higher temperature, experience a force in the direction opposite to that of the gradient. This phenomenon is known as thermophoresis. As the droplets can be sub-micron sized, the effects of brownian motion is also included. The lift force on the droplet due to shear, called as Saffman's lift force is also considered. The details related to modelling of these forces can be obtained in [14].

Theoretically, evaporation occurs at two stages: (i) When temperature is higher than the saturation temperature based on local water vapor concentration, water evaporates and the evaporation is controlled by the water vapor partial pressure until 100\% relative humidity is achieved; and when (ii) the boiling temperature (determined by the air-water mixture pressure) is reached, water continues to evaporate. After the droplet is evaporated due to either high-temperature or lowmoisture partial pressure, the vapour diffuses into the main flow and is transported away. The rate of vaporization is governed by the concentration difference between the surface and the airstream, and the corresponding mass change rate of the droplet can be given by

$$
\frac{d m_{p}}{d t}=\pi d^{2} k_{c}\left(C_{s}-C_{\infty}\right)
$$

where $k_{c}$ is the mass transfer coefficient and $C_{s}$ is the concentration of the vapor at the droplet surface, which is evaluated by assuming that the flow over the surface is saturated. $C_{\infty}$ is the vapor concentration of the bulk flow, obtained by solving the transport equations. The values of $k_{c}$ can be calculated from empirical correlations for $S h$ as mentioned in earlier works [13]. When the droplet temperature reaches the boiling point, the following equation can be used to evaluate its evaporation rate 


$$
\frac{d m_{p}}{d t}=\pi d^{2}\left(\frac{\lambda}{d}\right)\left(2+0.46 R e_{d}^{0.5}\right)\left(\frac{1}{C_{p}}\right) \ln \left(1+\frac{C_{p}\left(T_{\infty}-T\right)}{h_{f g}}\right)
$$

where $\lambda$ is the thermal conductivity of the gas/air and $h_{f g}$ is latent heat of water. $\mathrm{C}_{p}$ is the specific heat of the bulk flow. The droplet temperature can also be changed due to heat transfer between droplets and the continuous phase. Without considering radiation heat transfer, the droplet's sensible heat change depends on the convective heat transfer and latent heat $h_{f g}$, as shown in the following:

$$
m_{p} C_{p} \frac{d T}{d t}=\pi d^{2} h\left(T_{\infty}-T\right)+\frac{d m_{p}}{d t} h_{f g}
$$

where the convective heat transfer coefficient $h$ can be obtained with an empirical correlation of $N u$ as presented in earlier works [13].

The commercial software package FLUENT, from Fluent, Inc., version 17.2 [14] is used. The SIMPLE algorithm is used to couple the pressure and velocity. A second-order upwind scheme is used for spatial discretization of the convective terms and species. The Lagrangian trajectory calculations were employed to model the dispersed phase of droplets, including coupling with the continuous phase. The impact of the droplets on the continuous phase is considered as source terms to the governing equations. After obtaining an approximate flow field of the continuous phase - airflow in this study, FLUENT traces the droplet trajectories and computes heat and mass transfer between the droplets and the airflow.

\section{Boundary conditions and operating parameters}

The mainstream air velocity is $10 \mathrm{~m} / \mathrm{s}$ and temperature is $400 \mathrm{~K}$. The mainstream flow is assumed to be dry air with $0 \%$ humidity, and the jet flow is also dry air mixed with water droplets. The coolant jet velocity is also $10 \mathrm{~m} / \mathrm{s}$ and the temperature is $300 \mathrm{~K}$. The inlet conditions of the turbulence are $1 \mathrm{~m}^{2} / \mathrm{s}^{2}$ for the turbulence kinetic energy and $1 \mathrm{~m}^{2} / \mathrm{s}^{3}$ for the dissipation rate. The flow exit outlet of main computational domain is assumed to have a constant pressure. All the walls in the computational domain are adiabatic and have a nonslip boundary condition. The jet has a flow rate of $0.00035 \mathrm{~kg} / \mathrm{s}$ with $2 \%$ mist. Droplets of diameter $5 \mu \mathrm{m}$ and $10 \mu \mathrm{m}$ are considered. Various jet angles ranging from $15^{\circ}$ to $55^{\circ}$ are considered.

(a)

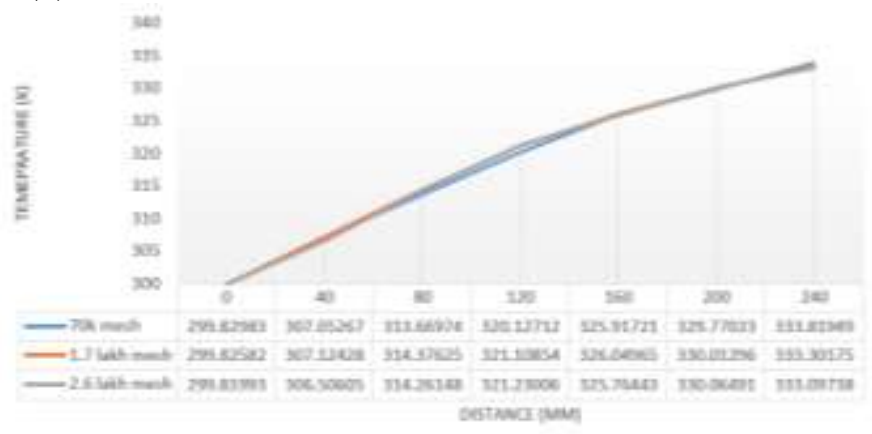

(b)

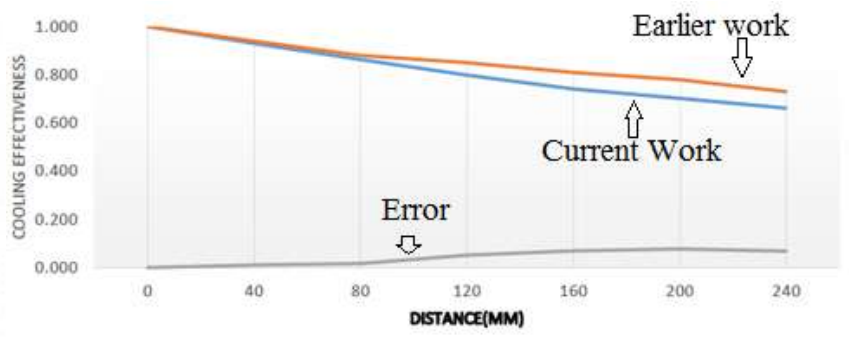

Fig. 2: (a) Grid independence tests in terms of temperature along the walls for various grid sizes and (b) comparison of the cooling effectiveness of as obtained from the current work with the earlier work [6] for droplet diameter, $10 \mu \mathrm{m}$ and inlet jet angle of $35^{\circ}$.

\section{Mesh and Validation}

An unstructured mesh is used to discretize the governing equations [Right panel of Fig. 1]. The meshing is adaptive with many refined cell elements near the coolant hole. The grid independence test is carried out to fix the number of nodes 
for the problem [see left panel of Fig. 2]. It was can be observed that there is no significant difference between 70,000 and 100,000 mesh. Therefore the mesh with 70,000 cells is used in the present study. The right panel of Fig. 2 illustrates the variation of the cooling effectiveness with the distance along the downstream region in the current work and earlier work [6] and the results are in excellent agreement. The error in the result might be due to the different mesh structures of the current work and earlier work. However, the current mesh is more efficient based on the refinement near the regions of higher gradients.

(a)

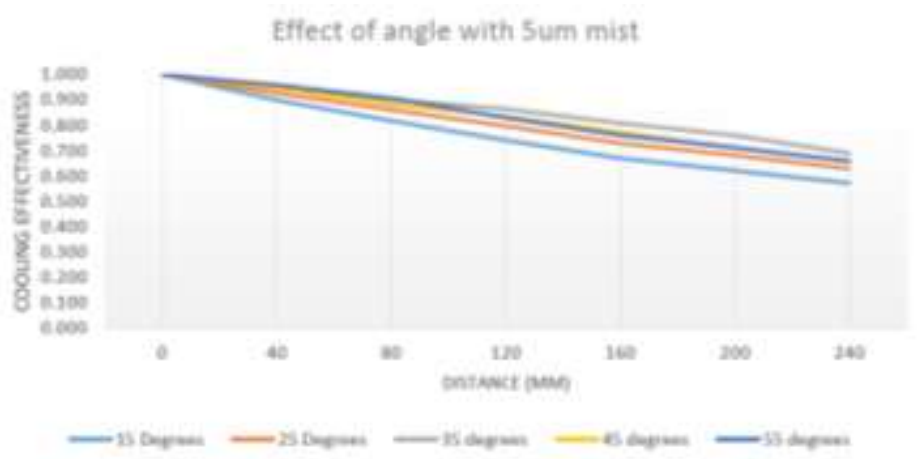

Fig. 3: Cooling Effectiveness along the distance for (a) droplets of $5 \mu \mathrm{m}$ diameter and (b) droplets of $10 \mu \mathrm{m}$ diameter at inlet jet angle of $35^{\circ}$.

\section{Results and Discussions}

The performance of mist assisted film cooling is studied by evaluating adiabatic cooling effectiveness $\eta$. It is defined as

$$
\eta=\frac{T_{g}-T_{a w}}{T_{g}-T_{c}}
$$

Where $\mathrm{T}_{\mathrm{g}}$ is main stream hot gas temperature, $\mathrm{T}_{\mathrm{c}}$ is temperature of coolant jet and $\mathrm{T}_{\mathrm{aw}}$ is adiabatic wall temperature. $\eta$ varies between 0 and 1 .

Figure 3(a) and (b) illustrate the cooling effectiveness along the length of the wall for various coolant inlet angles for droplet diameters $5 \mu \mathrm{m}$ and $10 \mu \mathrm{m}$, respectively. Common to all the angles and droplet diameters, the cooling effectiveness decreases with the distance along the downstream. The decrease of the effectiveness downstream of the hole is attributed to the fact that, the coolant gains heat from the mainstream. Overall, the largest cooling effectiveness is observed for the angle of $35^{\circ}$ involving both the droplet diameters. As seen from Fig. 3(a) the largest value of cooling effectiveness is observed for $35^{\circ}$ along major portion of the downstream for $5 \mu \mathrm{m}$ whereas, the effectiveness is largest along the entire length of the downstream for $35^{\circ}$ involving $10 \mu \mathrm{m}$ droplet diameters. As seen from Figures 3(a) and (b), the cooling effectiveness decreases at a slightly lesser rate for smaller droplets compared to the larger droplets. Intuitively it is expected that cooling effectiveness for $5 \mu \mathrm{m}$ droplets is higher than $10 \mu \mathrm{m}$ droplets due to higher rate of droplet evaporation. But the difference is not significant in the present study, as droplets in both the cases are expected to be evaporated completely before crossing the outlet. This can be witnessed from following expression for approximate droplet evaporation rate (from [5])

$$
\frac{h_{f g} \rho d^{2}}{2 \lambda\left(T_{\infty}-T\right)}
$$


where $h_{f g}$ is droplet latent heat and $\lambda$ is heat conductivity of air. For a droplet of $10 \mu m$ diameter and a temperature difference of $100 \mathrm{~K}$, the evaporation time is $0.038 \mathrm{~s}$. Typical average droplet velocity in current simulations is around 6 $\mathrm{m} / \mathrm{s}$. At this condition, droplets less than $10 \mu \mathrm{m}$ diameter are expected to evaporate with in the downstream length of the coolant jet slot (which is $0.24 \mathrm{~m}$ ).

The temperature contours for different coolant hole angles and droplet diameters are also plotted [Figures not shown for brevity]. The isotherms shows the gradient of temperature of the film [see Fig. 4]. The film temperature is lowest near the coolant hole that further increases and merges with the mainstream flow. Also, the thickness of the film in the vertical direction gradually increases in the downstream region for all angles and droplet diameters. The comparison between two droplet diameters depicts that, the temperature of the film is less as the jet leaves the hole for both the droplet diameters. However, for the smaller diameter, the vertical thickness as well as the length of the cold region is larger as seen from Fig. 4(a). This is due to the fact that, the smaller droplets results in more number of droplets for a fixed mass flow rate resulting in larger evaporation area. Also, as explained earlier, the cooling effectiveness on the wall is larger for the case with smaller diameters.

(a)

(b)
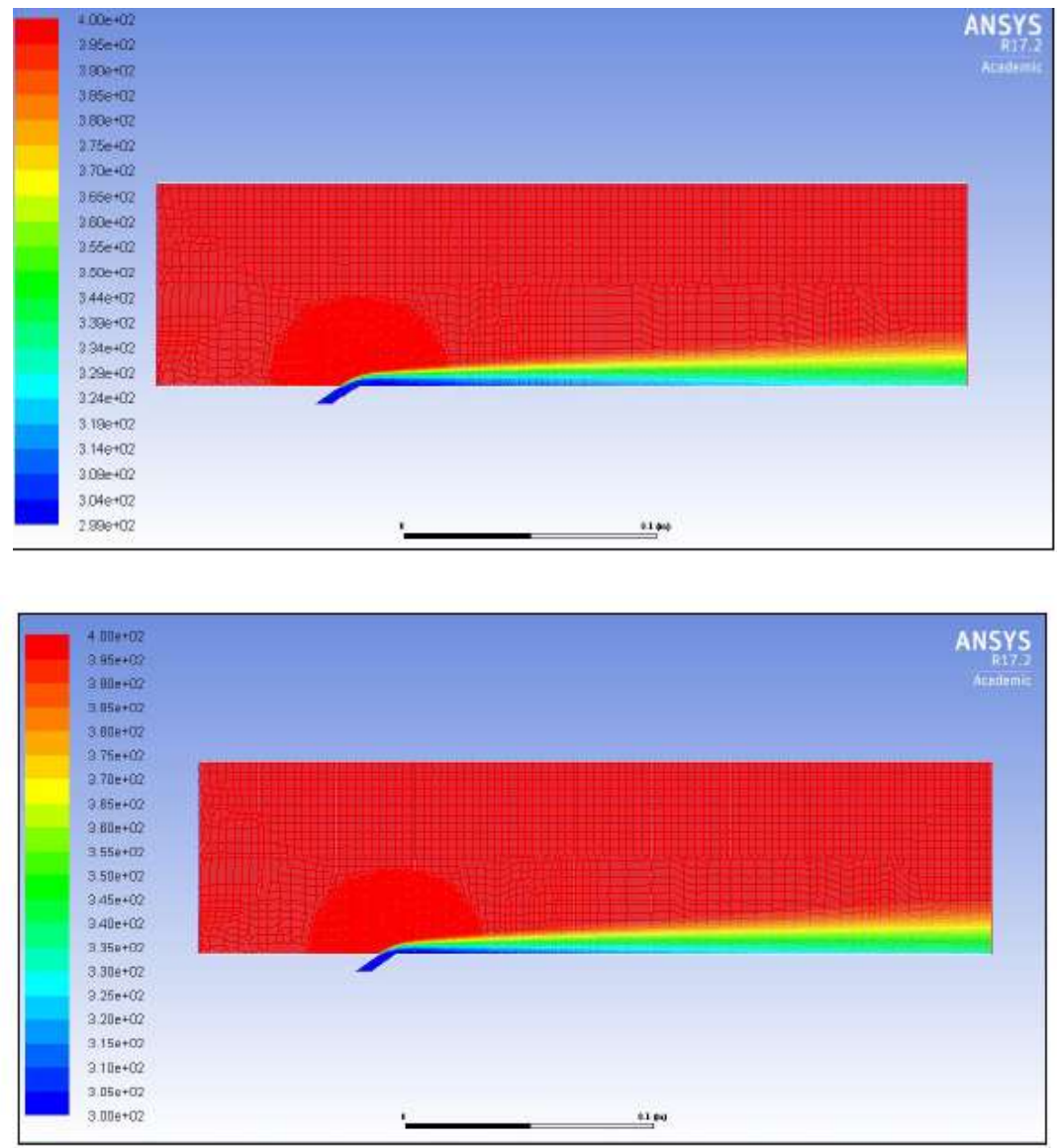

Fig. 4: Temperature contours for droplets of (a) $5 \mu \mathrm{m}$ and (b) $10 \mu \mathrm{m}$ diameter and inlet jet angle of $35^{\circ}$.

As seen from the previous results, the droplets are evaporating and the coolant air is becoming hotter in the downstream region. Thereafter, the cooling film disappears as the coolant fluid mixes with the mainstream fluid. To avoid this problem, the performance of film cooling based on two film cooling holes are also studied. Another film cooling hole is carefully located along the downstream where the coolant fluid tends to mix with the mainstream fluid. 
Fig. 5 illustrates the isotherms and comparison of cooling effectiveness for the single and double hole cases for mist assisted film cooling.

(a)

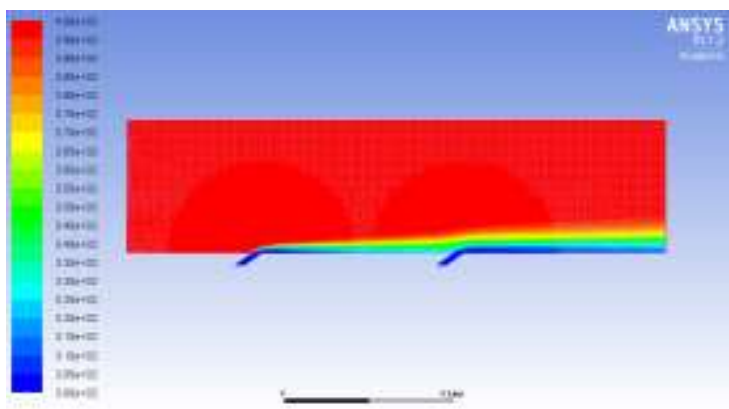

(b)

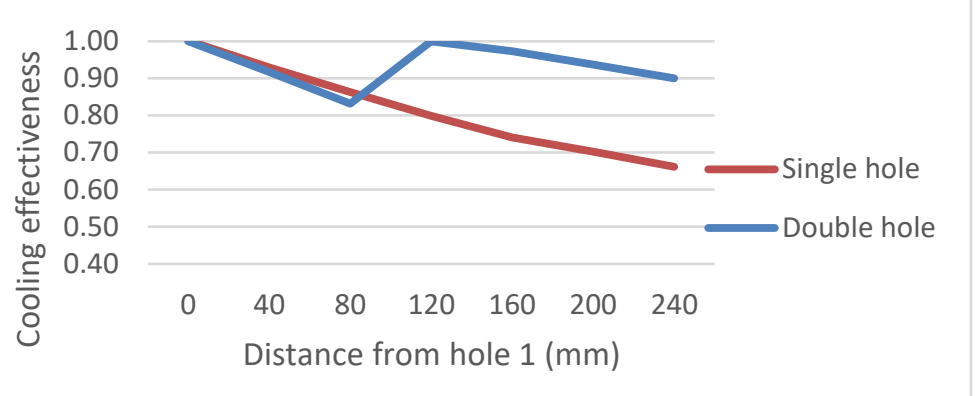

Fig. 5: (a) Isotherms for mist assisted film cooling with two holes and (b) comparison of cooling effectiveness for single hole and two holes for mist of with 35 degrees inlet angle.

\section{Conclusion}

The concept of mist assisted film cooling, involving the evaporation of water droplets is studied. The film cooling of a flat plate is considered where small water droplets in the form of mist are injected together with the coolant air jet. Various interesting trends on the effects of droplet diameter and coolant jet angles are observed. Overall, the cooling effectiveness is found to be larger for the mist assisted cases compared to the dry air case. This is due to the cooling experienced due to the evaporation of the droplets along the downstream. The diameter of droplets and angle of injection are two crucial parameters and the effects of these parameters on the cooling effectiveness are significant. Overall, the better cooling effectiveness values are observed for smaller droplets at specified mist mass concentration. The optimized angle for the operation is found as $35^{\circ}$ for the considered cases. The introduction of second coolant hole affects the cooling effectiveness significantly. The present study would be extended to predict performance of mist assisted film cooling using three-dimensional simulations.

\section{References}

[1] T. Guo, T. Wang, J. L. Gaddis, "Mist/Steam Cooling in a Heated Horizontal Tube Part I: Experimental System," ASME J. of Turbomachinery, vol. 122, pp. 360-365, 2000.

[2] T. Guo, T. Wang, J. L. Gaddis, "Mist/steam cooling in a heated horizontal tube - Part 2: Results and modelling," J. Turbomachinary-Trans. ASME, vol. 122, pp. 366-374, 2000.

[3] T. Guo, T. Wang, J. L. Gaddis, "Mist/Steam Cooling in a 180-Degree Tube," ASME J. Heat Transfer, vol. 122, pp. 749$756,2000$.

[4] X. S. Wang, G. X. Liao, W. C. Fan, R. Dobashi, "Experimental study on cooling a hot solid surface with water mist," J. Fire Sciences, vol. 22, pp. 355-366, 2004.

[5] X. Li, T. Wang, "Simulation of film cooling enhancement with mist injection," ASME J. Heat Transfer, vol. 128, pp. 509-519, 2006.

[6] X. Li, T. Wang, "Effects of various modeling on mist film cooling," ASME J. Heat Transfer, vol. 129, pp. 472-482, 2007.

[7] X. Li, T. Wang, "Two-phase ow simulation of mist film cooling on turbine blades with conjugate internal cooling," $J$. Heat Transfer, vol. 130, Art. no. 102901, 2008.

[8] T. Wang X. Li, "Mist film cooling simulation at gas turbine operating conditions," Int. J. Heat Mass Transfer, vol. 51, pp. 5305-5317, 2008. 
[9] K. H. Kim, H. J. Ko, K. Kim, H. Perez-Blanco, "Analysis of water droplet evaporation in a gas turbine inlet fogging process," Appl. Thermal Engn. Vol. 33-34, pp. 62-69, 2012.

[10] T. S. Dhanasekaran, T. Wang, "Numerical model validation and prediction of mist/steam cooling in a 180-degree bend tube," Int. J. Heat Mass Transfer, vol. 55, pp. 3818-3828, 2012.

[11] T. S. Dhanasekaran, T. Wang, "Computational analysis of mist/air cooling in a two-pass rectangular rotating channel with 45-deg angled rib turbulators," Int. J. Heat Mass Transfer, vol. 61, pp. 554-564, 2013.

[12] Y. Jiang, Q. Zheng, P. Dong, J. Yao, H. Zhang, J. Gao, "Conjugate heat transfer analysis of leading edge and downstream mist-air film cooling on turbine vane," Int. J. Heat Mass Transfer, vol. 90, pp. 613-626, 2015.

[13] W. E. Ranz, W. R. Marshall Jr., "Evaporation From Drops, Part I," Chem. Eng. Prog., vol. 48, pp. 141-146, 1952.

[14] Fluent, FLUENT 17.2 User's Guide FLUENT, Inc., Lebanon: NH (2017). 